\title{
2018 Year in Review: Adult Invasive Mechanical Ventilation
}

\author{
Karsten J Roberts
}

\author{
Introduction \\ Ventilatory Support \\ Patients Without ARDS \\ COPD \\ Hyperoxia \\ Ventilator-Associated Events \\ Effects in Prolonged Weaning \\ Prevention of Events \\ Ventilator Asynchrony \\ Sedation \\ Frequency of Asynchrony \\ Ventilator Liberation \\ SBT and Sedation Protocol in ARDS \\ 30-min Versus 120-min SBT \\ Extubation to Noninvasive Ventilation \\ Summary
}

\begin{abstract}
Research in the area of adult invasive mechanical ventilation is rich and diverse. With more than 3,200 articles on mechanical ventilation published in 2018, isolating the most relevant literature is a challenge. Separated into 5 themes (ie, ventilatory support, hyperoxia, ventilator-associated events, prevention of events, and ventilator liberation), this article will describe the most important papers published on adult invasive mechanical ventilation in 2018. Key words: mechanical ventilation; ventilatory support; hyperoxia; ventilator asynchrony; ventilator-associated events; ventilator liberation. [Respir Care 2019;64(5):604-609. (C) 2019 Daedalus Enterprises]
\end{abstract}

\section{Introduction}

Patients receiving invasive mechanical ventilation report feelings of pain, loneliness, discomfort, terror, and anxiety. ${ }^{1,2}$ Mitigation of these adverse events are common challenges for the ICU team. Improving synchrony, opti-

Mr Roberts is affiliated with the Hospital of the University of Pennsylvania, Department of Respiratory Care Services, Philadelphia, Pennsylvania.

Mr Roberts presented a version of this paper at the Year in Review of the AARC Congress 2018, held December 4-7, 2018, in Las Vegas, Nevada.

The author has disclosed no conflicts of interest. mizing sedation, early mobility, and prevention of complications are all goals in the evidence based management of the ventilated patient.

There are 5 prevalent themes in recently published literature on mechanical ventilation. As they relate to an imagined wish list for mechanical ventilation, the themes are ventilator support and hyperoxia (with the aim to pre-

Correspondence: Karsten J Roberts MSc RRT, Hospital of the University of Pennsylvania, Department of Respiratory Care Services, 3400 Spruce Street, Ground Floor, Founders Building, Philadelphia, PA 19104. E-mail: karsten.j.roberts@gmail.com.

DOI: $10.4187 /$ respcare. 06927 
vent injury), ventilator-associated events and ventilator asynchrony (with the aim to prevent iatrogenic complications), and ventilator liberation (with the aim to liberate the patient from the ventilator as soon as possible). Research in the area of adult invasive mechanical ventilation is rich and diverse. With more than 3,200 articles published in 2018, it is a challenge to isolate the most relevant literature published in the last calendar year. With these themes in mind, this article will review the pertinent literature published on adult invasive mechanical ventilation in 2018 .

\section{Ventilatory Support}

\section{Patients Without ARDS}

The last 2 decades have provided a plethora of data supporting low tidal volume $\left(\mathrm{V}_{\mathrm{T}}\right)$ in patients with ARDS. . $^{3-6}$ Plausible benefits of using low $\mathrm{V}_{\mathrm{T}}$ in patients without ARDS are fewer complications and decreased ventilator days. ${ }^{7}$ To investigate the potential effectiveness of low $V_{T}$ versus intermediate $\mathrm{V}_{\mathrm{T}}$ on ventilator-free days, the Protective Ventilation in Patients Without ARDS (PReVENT) trial was conducted. ${ }^{7}$

Designed as a randomized control trial, the primary outcome of the PReVENT trial was ventilator-free days at day 28 in subjects without ARDS. ${ }^{7} \mathrm{~A}$ low- $\mathrm{V}_{\mathrm{T}}$ strategy was initiated in 475 subjects and defined as $6 \mathrm{~mL} / \mathrm{kg}$ of predicted body weight. Comparatively, 480 individuals were assigned to a group with an "intermediate" $\mathrm{V}_{\mathrm{T}}$ of $10 \mathrm{~mL} / \mathrm{kg}$ predicted body weight. Both groups had 21 (mean) ventilator free days with no significant differences in ICU length of stay (mean 6 vs 6 d) or hospital length of stay (14 vs $15 \mathrm{~d}$ ). Other high-value outcomes of 28-d and 90-d mortality showed no significant differences between groups. There were no significant differences in adverse events (eg, development of ARDS, pneumonia, severe atelectasis, or pneumothorax) between groups. ${ }^{7}$

Recent post hoc observational analysis of previous ARDS trials completed found that decreased driving pressure $(\Delta \mathrm{P})$ is associated with increased survival. ${ }^{8}$ Schmidt et al ${ }^{9}$ shifted the focus on $\Delta \mathrm{P}$ toward subjects without ARDS. A retrospective study of 622 subjects was conducted to determine association of $\Delta \mathrm{P}$ with mortality on the first day of mechanical ventilation. Classification of non-ARDS versus ARDS was tested using the same model as Amato et al. ${ }^{8}$ Similarly, to confirm the accuracy of their analysis model, Schmidt et al $^{9}$ validated data in 543 subjects. The study confirmed an association between $\Delta \mathrm{P}$ and mortality in ARDS. However, an independent association between $\triangle \mathrm{P}$ and mortality in subjects without ARDS was not established. This was further confirmed by a lack of association with secondary outcomes of hospital, ICU, and 6-month mortality. ${ }^{9}$

\section{COPD}

Due to reportedly higher mortality in subjects with COPD exacerbations, clinicians avoid invasive mechanical ventilation and opt for noninvasive ventilation. A retrospective study by Gadre et al ${ }^{10}$ included subjects with severe COPD and respiratory failure and sought to describe characteristics and outcomes in subjects with COPD who received invasive mechanical ventilation. Baseline diagnosis of COPD according to the Global Initiative for Obstructive Lung Diseases standard was confirmed using pre-hospital pulmonary function tests. A total of 670 subjects with a pre-morbid diagnosis of COPD were included in the study, $88 \%$ of whom were intubated for other etiologies, most commonly pneumonia.

Overall hospital mortality was $25 \%$, with mortality being significantly lower in subjects with COPD exacerbations without additional comorbidities. Subjects with primary COPD exacerbations were more likely to be admitted directly to the ICU from the emergency department. Median duration of mechanical ventilation was $3 \mathrm{~d}$, median ICU length of stay was $5 \mathrm{~d}$, and median hospital length of stay was $12 \mathrm{~d}$. ICU mortality and hospital mortality were lower in subjects with COPD exacerbations. Twenty-six percent of the subjects were readmitted and mechanically ventilated within the 4-y study period. Shorter durations of mechanical ventilation and discharges to home were more prevalent in subjects without comorbidities. Discharges to home were also associated with overall improved survival. ${ }^{10}$

The application of mechanical ventilation in any disease process is complicated and unique. The fact that patients without ARDS had no significant differences in clinical outcomes raises new questions about what variables may influence duration of mechanical ventilation when using a low- $\mathrm{V}_{\mathrm{T}}$ strategy. As our understanding of a low- $\mathrm{V}_{\mathrm{T}}$ strategy continues to evolve, more research will be needed, not only in subjects with or without ARDS, but also in patients with other etiologies. The use of low- $\mathrm{V}_{\mathrm{T}}$ strategies in COPD, for example, may lead to improved outcomes in a patient population that continues to increase. Similarly, future research may elucidate the role of $\Delta \mathrm{P}$ in subjects with etiologies other than ARDS.

\section{Hyperoxia}

The detrimental effects of oxygen on the lungs has been known for more than a century. ${ }^{11}$ Harm caused by hyperoxia may not only resemble ARDS, but it may also do more lung damage in patients with ARDS. ${ }^{11,12}$ Two recent studies have reported hyperoxia to be common in mechanically ventilated subjects. ${ }^{12,13}$

Aggarwal et al ${ }^{12}$ extracted data from several ARDSNet studies and reviewed cases with a $\mathrm{P}_{\mathrm{aO}_{2}}>80 \mathrm{~mm} \mathrm{Hg}$. Hyperoxia, or above-goal oxygen exposure, was defined 
as a difference between set $\mathrm{F}_{\mathrm{IO}_{2}}$ and $0.5 \mathrm{~F}_{\mathrm{IO}_{2}}$ when $\mathrm{F}_{\mathrm{IO}_{2}}$ was $>0.5$ and $\mathrm{P}_{\mathrm{aO}_{2}}$ was $>80 \mathrm{~mm} \mathrm{Hg}$. Criteria for hyperoxia was met in 2,994 subjects who had a mean excess $\mathrm{F}_{\mathrm{IO}_{2}}$ of 0.24 . In this population, mortality was higher and subjects had fewer ventilator-free days than those with normoxia. Furthermore, fewer hospital-free days were reported, and the authors concluded that high $\mathrm{O}_{2}$ exposure was associated with worse overall clinical outcomes. ${ }^{12}$

A second observational study regarding hyperoxia in mechanically ventilated subjects was performed in Japan by Egi et al. ${ }^{13}$ The aim of the study was not only to observe the relationship of $\mathrm{F}_{\mathrm{IO}_{2}}$ and $\mathrm{P}_{\mathrm{aO}}$, but also to observe how $\mathrm{F}_{\mathrm{IO}_{2}}$ was managed by clinicians in the setting of hyperoxia. The study included 454 subjects from 28 ICUs who were intubated and mechanically ventilated for $>48 \mathrm{~h}$.

Subjects had $\mathrm{F}_{\mathrm{IO}_{2}}$ set at $0.3-0.49$ for the majority of their ventilator course. With a median $\mathrm{P}_{\mathrm{aO}_{2}}$ equal to $90 \mathrm{~mm} \mathrm{Hg}, 47 \%$ of subjects had $\mathrm{P}_{\mathrm{aO}_{2}}>100 \mathrm{~mm} \mathrm{Hg}$ during the study. ${ }^{13}$

Hyperoxia and the resulting ill-effects are clearly not conjectural in nature. These studies give ICU clinicians guidance on how to better manage oxygenation in critically ill patients. These data may also lead to future changes in clinical practice guides regarding the oxygen management in mechanically ventilated populations.

\section{Ventilator-Associated Events}

\section{Effects in or on Prolonged Weaning}

In 2013, the Centers for Disease Control and Prevention (CDC) published supportive literature to better define ventilator-associated events (VAEs). ${ }^{14}$ This has led to an increase in publications related to identification, prevention, and treatment of ventilator-associated conditions (VACs), infectious VACs (IVACs), and ventilator-associated pneumonia (VAP), three mutually exclusive conditions under the category of VAEs. ${ }^{15-18}$

A single-center, retrospective cohort conducted by Kobayashi et al ${ }^{19}$ enrolled 404 subjects to determine clinical outcomes in patients with VACs, IVACs, and VAP. Subjects enrolled had been mechanically ventilated for at least $4 \mathrm{~d}$. Subjects were categorized as IVAC, non-IVAC, or non-VAC. Study results reported 54 subjects with VACs and 23 with IVACs. Twenty-one subjects were diagnosed with VAP. These conditions developed a mean $9.2 \mathrm{~d}$ from initiation of mechanical ventilation, with a median of $4.5 \mathrm{~d}$. Median days on invasive mechanical ventilation and ICU stay were $7 \mathrm{~d}$ and $11 \mathrm{~d}$, respectively. Hospital mortality was independently associated with IVACs, whereas VAP was not associated with hospital death. VACs had a similar association, but it was not statistically significant. The study concluded that VAP does not increase mortality, but VACs and IVACs do increase mortality.

\section{Prevention of Events}

Monitoring patient cuff pressures is necessary to prevent aspiration of secretions. ${ }^{20}$ There is, however, no accepted standard of practice for the frequency of monitoring cuff pressures. Letvin et $\mathrm{al}^{21}$ randomized subjects to frequent monitoring or infrequent monitoring to determine non-inferiority. A total of 166 subjects were randomized to frequent monitoring, defined as after intubation, regardless of location (ICU vs hospital ward); upon admission to the ICU, every $8 \mathrm{~h}$; and when clinically indicated (eg, cuff leaks). Infrequent monitoring $(n=139)$ was carried out only every $8 \mathrm{~h}$ and when clinically indicated. In total, 1,531 cuff pressures were recorded in the frequent group, and 336 cuff pressures were recorded in the infrequent monitoring group. VAEs were monitored for occurrence and frequency. Secondary outcomes included 30-d and 100-d mortality, length of hospital stay, witnessed aspiration events, and VAP rates, and 30-d readmissions. ${ }^{21}$

The number of VAEs observed was not found to be statistically significant. Similarly, the number of witnessed aspirations and VAP rates were the same in both groups. There was no statistical significance found for 30-d mortality or hospital stay. Only 30-d hospital readmission was found to have a statistically significant difference between groups. As a result of these findings, the authors concluded that more frequent cuff endotracheal tube cuff monitoring was not associated with any outcome benefits. ${ }^{21}$

When an agency such as the CDC changes recommendations or guidelines, there is a reasonable chance that new research will ensue. Publication of clinical outcomes in patients presenting with VAEs, with or without VAP, will most certainly lead to a better understanding of how to prevent such events. Although not all interventions have outcome benefits, studies of clinical interventions with potential benefits help narrow the focus on what interventions should be changed in the clinical management of invasively ventilated patients.

\section{Ventilator Asynchrony}

\section{Sedation}

Ventilator-induced lung injury (VILI) can be caused by ventilator asynchrony. ${ }^{22}$ Using pressure-time, flow-time, and volume-time data, Sottile et al $^{22}$ developed machine learning algorithms to detect 4 types of ventilator asynchrony. To determine the association between ventilator asynchrony and sedation, 4.26 million breaths were analyzed. Thirty-four percent of the breaths measured were determined to be asynchronous. $\mathrm{V}_{\mathrm{T}}>10 \mathrm{~mL} / \mathrm{kg}$ predicted 
body weight were found in double-triggered and flowlimited breath profiles. In addition, although deep sedation was found to reduce the frequency of asynchronous breaths, only neuromuscular blockade eliminated ventilator asynchrony. ${ }^{22}$

This prospective cohort study described an exciting area of study in ventilator asynchrony in patients at risk for ARDS. ${ }^{22}$ The authors added to previously described data that used automated systems to detect asynchrony. However, this is the first time that accuracy, sensitivity, and specificity were tested. Time will tell if artificial intelligence and clinicians' ability to identify unsafe conditions via remote clinical applications could lead to greater patient safety.

\section{Frequency of Asynchrony}

Recognizing that double-triggered breaths lead to greater than expected $\mathrm{V}_{\mathrm{T}}$, de Haro et $\mathrm{al}^{23}$ analyzed the incidence, mechanism, and physiology of double-triggered breaths. This prospective, observational study continuously monitored 67 subjects and collected a total of 9.69 million breaths. Although double-triggering occurred in just $0.6 \%$ of the breaths analyzed, all of the subjects included in the study exhibited double-triggering. Interestingly, doubletriggering occurred more frequently in pressure-controlled ventilation than in volume controlled ventilation. This was true regardless of volume controlled breaths with or without decelerating flow. Despite these findings, $\mathrm{V}_{\mathrm{T}}$ values were higher in volume controlled ventilation with constant flow and with decelerating flow patterns than in pressure controlled breaths. ${ }^{23}$

The data from these articles emphasize the importance of vigilance when observing patients for double-triggering and ventilator asynchrony. The door has been opened for developing more research regarding artificial intelligence and machine learning. Larger-scale research may lead to further understanding about ventilator asynchrony and clinicians' ability to apply machine learning in an ICU environment. Furthermore, having a deeper understanding of patient interaction with mechanical ventilation may guide research concerning timing of, and patient response to, pharmacologic interventions. ${ }^{22}$

\section{Ventilator Liberation}

Although mechanical ventilation is necessary supportive care, the goal is safe and effective liberation. ${ }^{24}$ Spontaneous breathing in concert with daily sedation interruption have been shown to decrease the duration of mechanical ventilation. ${ }^{24,25}$ Length of spontaneous breathing trials (SBT), the use of noninvasive ventilation, and the use of conservative sedation were recently studied. ${ }^{25-27}$

\section{SBT and Sedation Protocol in ARDS}

Kallet et $\mathrm{a}^{25}$ investigated the reduction of mechanical ventilation days in ARDS after the implementation of an SBT and sedation protocol. The authors compared 397 preprotocol subjects and 656 post-protocol subjects. Patients with ARDS who were managed with the protocol saw a significant decrease in the number of ventilator days. Similarly, a statistically significant decrease in ICU days was seen after the protocol's implementation. A median of 2 SBTs were needed for subjects to achieve ventilator liberation. Improved outcomes were also associated with higher pulmonary compliance in patients managed with the SBT and sedation-reduction protocol. These results show that, despite longer duration of mechanical ventilation, subjects with ARDS were not as difficult to liberate from mechanical ventilation as one might assume (Fig. 1). ${ }^{25}$

\section{0-min Versus 120-min SBT}

Pressure support ventilation used to perform a 30-min SBT may overestimate a patient's ability to spontaneously breathe. Liang et $\mathrm{al}^{26}$ described this in a prospective study of 352 ICU subjects. All of the subjects passed a 30-min SBT prior to moving to a $120-\mathrm{min}$ SBT; 311 subjects successfully passed the 120-min SBT, whereas 41 subjects failed. Physiologic data revealed that subjects in the failure group had a higher heart rate and higher $\mathrm{P}_{\mathrm{aCO}}$ during the 30-min SBT than subjects in the success group. Subjects who failed the 120-min SBT also had higher heart rates, breathing frequencies, $\mathrm{P}_{\mathrm{aCO}}$, and rapid shallow breathing index after passing the 30-min SBT. Demographic data revealed that subjects in the failure group were older, had longer durations of mechanical ventilation, and underwent more (unsuccessful) trials before passing the SBT.

For statistically significant findings, the authors applied a point-based system to create decision trees for clinicians to determine whether patients should move from a 30-min SBT to a 120-min SBT. For each of the 4 physiologic parameters that had statistical significance, points were applied to help determine overall success or failure of the SBT. Subjects in the 30-min SBT group with $\leq 2$ points failed at a lower rate than subjects with $>4$ points. In the 120-min SBT, group the 5 physiological parameters with statistical significance were used for a similar decisionmaking tree. Like the 30-min study group, subjects with $\leq 2$ points had a low failure rate. Subjects in the 120-min group only needed a score of $>2$ points to identify failure. These findings reflected a great risk of overall failure in the 120-min SBT group. ${ }^{26}$ 


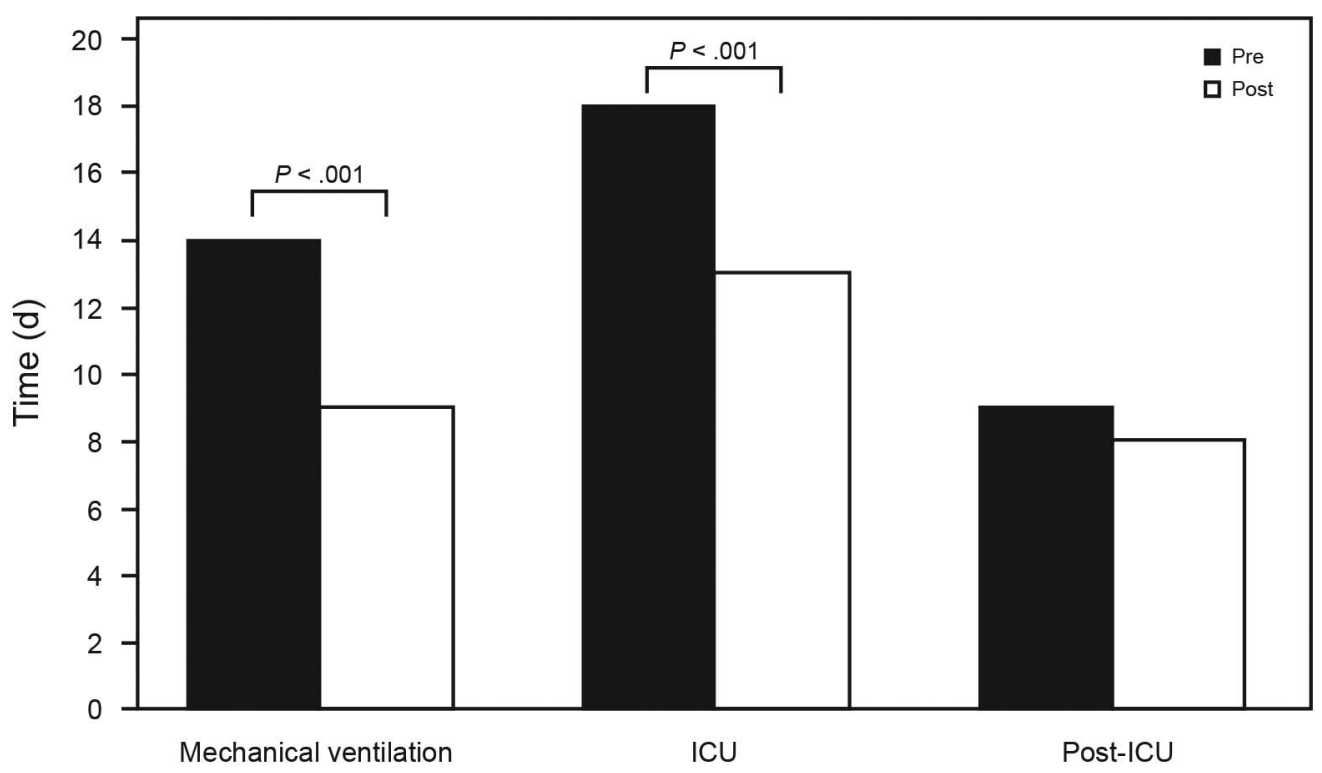

Fig. 1. Differences in median duration of mechanical ventilation, ICU length of stay, and post-ICU length of stay between the pre-protocol group and the post-protocol group whose weaning and sedation were governed by spontaneous breathing trials and daily sedation interruptions. From Reference 25.

\section{Extubation to Noninvasive Ventilation}

Some patients experience difficulty weaning from invasive mechanical ventilation. ${ }^{24}$ Perkins et $\mathrm{al}^{27}$ randomized subjects to either protocolized weaning and extubations to noninvasive ventilation or standard invasive weaning to determine effectiveness. Time from randomization to ventilator liberation, both invasive and noninvasive, was the primary outcome. Secondary outcomes included duration of invasive mechanical ventilation, re-intubation or tracheostomy, and survival.

Extubations to noninvasive ventilation did not shorten the time of liberation from all types of mechanical ventilation. ${ }^{27}$ Liberation from all forms of ventilation was $4.3 \mathrm{~d}$ in the noninvasive group versus $4.5 \mathrm{~d}$ in the invasively weaned group. For subjects extubated to noninvasive ventilation, less time was spent invasively ventilated overall ( $1 \mathrm{~d}$ vs $4 \mathrm{~d})$. The total number of ventilator days was not different between groups ( $3 \mathrm{~d}$ vs $4 \mathrm{~d}$ ). Re-intubations, tracheostomies, adverse events, and survival were all similar between the study groups. ${ }^{27}$

Liberation from all forms mechanical ventilation is the ultimate goal of supporting subjects through their disease process. Regardless of etiology, all subjects who meet clinical criteria should have daily interruptions to sedation and SBTs initiated. ${ }^{24}$ Although some subjects may require longer duration of mechanical ventilation, these concepts can safely and effectively be applied across disease processes. Despite removal from invasive mechanical ventilation being a theme of this review, subjects should also be liberated from noninvasive ventilation in a timely manner. ${ }^{27}$
Future epidemiologic research may provide further insight into how outcomes and ventilator-free days may be improved.

\section{Summary}

Research in the area of adult invasive mechanical ventilation is rich and diverse. The studies summarized here represent a small portion of the publications from 2018. The purpose of this review was to highlight the most relevant publications in the last calendar year. The themes found in these articles help organize relevant publications and summarize the findings.

\section{REFERENCES}

1. Rotondi AJ, Chelluri L, Sirio C, Mendelsohn A, Schulz R, Belle S, et al. Patients' recollections of stressful experiences while receiving prolonged mechanical ventilation in an intensive care unit. Crit Care Med 2002;30(4):746-752.

2. Chahraoui K, Laurent A, Bioy A, Quenot J-P. Psychological experience of patients 3 months after a stay in the intensive care unit: a descriptive and qualitative study. J Crit Care 2015;30(3):599-605.

3. Brower RG, Matthay MA, Morris A, Schoenfeld D, Thompson BT, Wheeler A. Ventilation with lower tidal volumes as compared with traditional tidal volumes for acute lung injury and the acute respiratory distress syndrome. N Engl J Med 2000;342(18):1301-1308.

4. Fan E, Needham DM, Stewart TE. Ventilatory management of acute lung injury and acute respiratory distress syndrome. JAMA 2005; 294(22):2889-2896.

5. Putensen C, Theuerkauf N, Zinserling J, Wrigge H, Pelosi P. Metaanalysis: ventilation strategies and outcomes of the acute respiratory distress syndrome and acute lung injury. Ann Intern Med 2009; 151(8):566-576. 
6. Needham DM, Yang T, Dinglas VD, Mendez-Tellez PA, Shanholtz C, Sevransky JE, et al. Timing of low tidal volume ventilation and intensive care unit mortality in acute respiratory distress syndrome. A prospective cohort study. Am J Respir Crit Care Med 2014;191(2): 177-185.

7. Writing Group for the PReVENT Investigators. Effect of a low vs intermediate tidal volume strategy on ventilator-free days in intensive care unit patients without ards: a randomized clinical trial. JAMA 2018;320(18):1872-1880.

8. Amato MBP, Meade MO, Slutsky AS, Brochard L, Costa ELV, Schoenfeld DA, et al. Driving pressure and survival in the acute respiratory distress syndrome. N Engl J Med 2015;372(8):747-755.

9. Schmidt MFS, Amaral ACKB, Fan E, Rubenfeld GD. Driving pressure and hospital mortality in patients without ARDS: a cohort study. Chest 2018;153(1):46-54.

10. Gadre SK, Duggal A, Mireles-Cabodevila E, Krishman S, Wang XF, Zell K, Guzman J. Acute respiratory failure requiring mechanical ventilation in severe chronic obstructive pulmonary disease (COPD). Medicine (Baltimore) 2018;97(17):e0487.

11. Martin DS, Grocott MPW. Oxygen therapy in critical illness: precise control of arterial oxygenation and permissive hypoxemia. Crit Care Med 2013;41(2):423-432.

12. Aggarwal NR, Brower RG, Hager DN, Thompson BT, Netzer G, Shanholtz C, et al. Oxygen exposure resulting in arterial oxygen tensions above the protocol goal was associated with worse clinical outcomes in acute respiratory distress syndrome. Crit Care Med 2018;46(4):517-524.

13. Egi M, Kataoka J, Ito T, Nishida O, Yasuda H, Okamaoto H, et al. Oxygen management in mechanically ventilated patients: a multicenter prospective observational study. J Crit Care 2018;46:1-5.

14. Magill SS, Klompas M, Balk R, Burns SM, Deutschman CS, Diekema $\mathrm{D}$, et al. Developing a new, national approach to surveillance for ventilator-associated events. Crit Care Med 2013;41(11):2467-2475.

15. Hayashi Y, Morisawa K, Klompas M, Jones M, Bandeshe H, Boots $\mathrm{R}$, et al. Toward improved surveillance: the impact of ventilatorassociated complications on length of stay and antibiotic use in patients in intensive care units. Clin Infect Dis Off Publ Infect Dis Soc Am 2013;56(4):471-477.

16. Klein Klouwenberg PMC, van Mourik MSM, Ong DSY, Horn J, Schultz MJ, Cremer OL, Bonten MJM. Electronic implementation of a novel surveillance paradigm for ventilator-associated events. Feasibility and validation. Am J Respir Crit Care Med 2014;189(8):947-955.
17. Klompas M, Anderson D, Trick W, Babcock H, Kerlin MP, Li L, et al. The preventability of ventilator-associated events. The CDC prevention epicenters wake up and breathe collaborative. Am J Respir Crit Care Med 2014;191(3):292-301.

18. Boyer AF, Schoenberg N, Babcock H, McMullen KM, Micek ST, Kollef MH. A prospective evaluation of ventilator-associated conditions and infection-related ventilator-associated conditions. Chest 2015;147(1):68-81.

19. Kobayashi H, Uchino S, Takinami M, Uezono S. The impact of ventilator-associated events in critically ill subjects with prolonged mechanical ventilation. Respir Care 2017;62(11):1379-1386.

20. Danielis M, Benatti S, Celotti P, De Monte A, Trombini O. Continuous monitoring of endotracheal tube cuff pressure: best practice in intensive care unit. Assist Inferm Ric 2015;34(1):15-20.

21. Letvin A, Kremer P, Silver PC, Samih N, Reed-Watts P, Kollef MH. Frequent versus infrequent monitoring of endotracheal tube cuff pressures. Respir Care 2018;63(5):495-501.

22. Sottile PD, Albers D, Higgins C, Mckeehan J, Moss MM. The association between ventilator asynchrony, delivered tidal volume, and sedation using a novel automated ventilator asynchrony detection algorithm. Crit Care Med 2018;46(2):e151-e157.

23. de Haro C, López-Aguilar J, Magrans R, Montanya J, FernándezGonzalo S, Turon M, et al. Double cycling during mechanical ventilation: frequency, mechanisms, and physiologic implications. Crit Care Med 2018;46(9):1385-1392.

24. Schmidt GA, Girard TD, Kress JP, Morris PE, Ouellette DR, Alhazzani $\mathrm{W}$, et al. Liberation from mechanical ventilation in critically ill adults: executive summary of an official American College of Chest Physicians/American Thoracic Society clinical practice guideline. Chest 2017;151(1):160-165.

25. Kallet RH, Zhuo H, Yip V, Gomez A, Lipnick MS. Spontaneous breathing trials and conservative sedation practices reduce mechanical ventilation duration in subjects with ARDS. Respir Care 2018; 63(1):1-10

26. Liang G, Liu T, Zeng Y, Shi Y, Yang W, Yang Y, Kang Y. Characteristics of subjects who failed a 120-minute spontaneous breathing trial. Respir Care 2018;63(4):388-394.

27. Perkins GD, Mistry D, Gates S, Goa F, Snelson C, Hart N, et al. Effect of protocolized weaning with early extubation to noninvasive ventilation vs invasive weaning on time to liberation from mechanical ventilation among patients with respiratory failure: the breathe randomized clinical trial. JAMA 2018;320(18):1881-1888. 